\title{
Voriconazole for chronic pulmonary aspergillosis: a prospective multicenter trial
}

\author{
J. Cadranel • B. Philippe • C. Hennequin • A. Bergeron • \\ E. Bergot • A. Bourdin • V. Cottin • T. Jeanfaivre • \\ C. Godet $\cdot$ M. Pineau $\cdot$ P. Germaud
}

Received: 28 March 2012 / Accepted: 21 June 2012 /Published online: 11 July 2012

(C) Springer-Verlag 2012

\begin{abstract}
Early evidence suggests the efficacy of voriconazole for chronic pulmonary aspergillosis (CPA). We conducted a prospective, open, multicenter trial to evaluate the efficacy and safety of voriconazole for proven CPA in minimally or non-immunocompromised patients. Patients had CPA confirmed by chest computed tomography (CT)
\end{abstract}

Electronic supplementary material The online version of this article (doi:10.1007/s10096-012-1690-y) contains supplementary material, which is available to authorized users.

J. Cadranel $(\triangle)$

Service de Pneumologie et Réanimation, AP-HP, Hôpital Tenon,

4 Rue de la Chine,

75020 Paris, France

e-mail: jacques.cadranel@tnn.aphp.fr

J. Cadranel

Université Pierre et Marie Curie-Paris 6,

4 Place Jussieu,

75005 Paris, France

\section{B. Philippe}

Service de Pneumologie, Hôpital René Dubos,

6 Avenue de Ile de France,

95300 Pontoise, France

C. Hennequin

INSERM, U945,

Paris, France

C. Hennequin

Université Pierre et Marie Curie_-Paris 6, UMR S945,

Paris, France

C. Hennequin

Service de Parasitologie-Mycologie, AP-HP, Hôpital St. Antoine,

Paris, France

\section{A. Bergeron}

Service de Pneumologie, AP-HP, Hop Saint Louis,

Université Paris Diderot, Sorbonne Paris-Cité,

75475 Paris, France and/or endoscopy, positive Aspergillus culture from a respiratory sample, and positive serologic test for Aspergillus precipitins. Patients received voriconazole (200 $\mathrm{mg}$ twice daily) for a period of 6-12 months and were followed for 6 months after the end of therapy (EOT). The primary endpoint was global success at 6 months, defined as

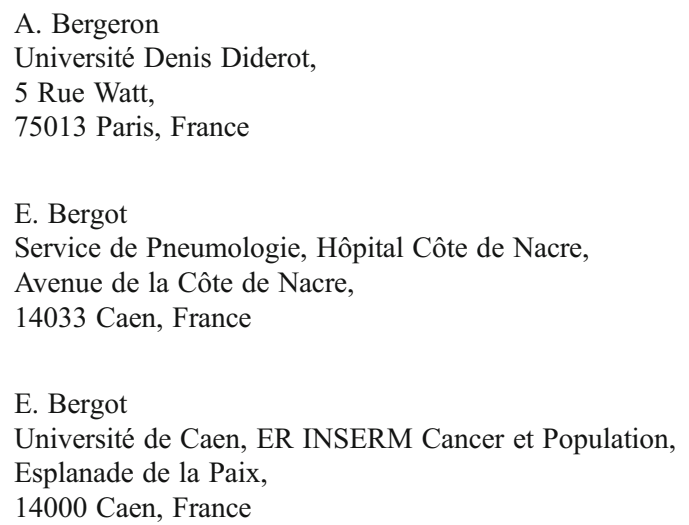

\section{A. Bourdin}

Department of Respiratory Disease,

Centre Hospitalier Universitaire,

371 Avenue du Doyen Giraud,

34090 Montpellier, France

\section{A. Bourdin}

Université Montpellier 1 et 2, INSERM U1046,

5 Boulevard Henri IV,

34000 Montpellier, France

V. Cottin

Hospices Civils de Lyon, Hôpital Louis Pradel,

28 Avenue Doyen Lepine,

69677 Bron, France 
complete or partial ( $\geq 50 \%$ improvement) radiological response and mycological eradication. Forty-one patients with confirmed CPA were enrolled. All patients had A. fumigatus as the etiologic agent. By EOT, five patients had died from comorbidities and seven had discontinued voriconazole due to toxicity. The global success rate at 6 months was $13 / 41$ (32\%): 10/19 (53\%) for chronic necrotizing aspergillosis and $3 / 22(14 \%)$ for chronic cavitary aspergillosis $(p=0.01)$. The respective success rates at EOT were 58 and $32 \%$. Clinical symptoms and quality of life also improved during treatment. Voriconazole is effective for CPA, with acceptable toxicity. The response rate is higher and obtained more rapidly in necrotizing than cavitary forms.

\section{Introduction}

The clinical spectrum of Aspergillus disease ranges from mild allergic manifestations to rapidly evolving invasive infections. While less studied, chronic pulmonary aspergillosis (CPA) seems to be more frequently encountered in patients with a history of lung disease.

As chronic disabling disease, CPA primarily affects middle-aged patients who have a multitude of underlying pulmonary conditions and who are not (or only minimally) immunosuppressed [1,2]. Advances in medical imaging have allowed better distinction between the different types of CPA, and, despite some overlap, classification into distinct entities is possible [1]: simple aspergilloma (fungus ball), chronic cavitary pulmonary aspergillosis (CCPA; also known as complex aspergilloma), chronic necrotizing pulmonary aspergillosis (CNPA; also known as semi-invasive

\author{
V. Cottin \\ Université Lyon I, UMR 754, \\ 43 Boulevard du 11 Novembre 1918 , \\ 69100 Villeurbanne, France \\ T. Jeanfaivre \\ Département de Pneumologie, Centre Hospitalier Universitaire, \\ 4 Rue Larrey, \\ 49933 Angers, France \\ C. Godet \\ Service des Maladies Infectieuses, Hôpital la Milétrie, \\ 2 Rue de la Miletrie, \\ 86021 Poitiers, France \\ M. Pineau \\ Pfizer Global Pharmaceuticals, \\ 23-25 Avenue du Dr Lannelongue, \\ 75668 Paris, France \\ P. Germaud \\ Service de Pneumologie, Institut du Thorax, Hôpital Laennec, \\ Boulevard Jacques Monod, \\ 44093 Nantes, France
}

or subacute invasive aspergillosis), chronic fibrosing pulmonary aspergillosis (an end result of untreated CCPA and, occasionally, also CNPA), and tracheobronchial aspergillosis (TBA) $[1,3,4]$.

CPA is associated with high morbidity and mortality $[3,5]$. Apart from simple aspergilloma, which can be treated surgically, antifungal therapy is usually the only treatment option. Intravenous amphotericin B has demonstrated some effectiveness in CNPA, but is often poorly tolerated and difficult to administer for sufficiently long durations [1]. Itraconazole has shown mixed results and a high relapse rate (over $20 \%$ ) [6]. Voriconazole, a second-generation triazole, is considered to be the treatment of choice for invasive aspergillosis. Indeed, it has been shown that the administration of voriconazole in any kind of therapeutic regimen is a significant prognostic factor for invasive infection [7-10]. In vitro, minimum inhibitory concentrations (MICs) against Aspergillus spp. are lower for voriconazole compared to itraconazole and amphotericin $\mathrm{B}$; in addition, voriconazole exhibits superior fungicidal activity against $A$. fumigatus, the leading cause of aspergillosis [11-13]. Voriconazole is rapidly absorbed with a high oral bioavailability of $90-96 \%$ in fasting conditions [12] and it penetrates well into tissues and body fluids, including the lung tissue and the pulmonary epithelial lining fluid [14].

Preliminary exploratory trials have shown voriconazole to be effective as primary or salvage therapy for CPA [15-17]. However, no prospective trials have been specifically designed to evaluate the efficacy of voriconazole in this setting. This multicenter, prospective trial was set up to evaluate, by means of a centralized review of clinical response and the use of predefined radiological criteria, the efficacy of voriconazole as a first-line therapy for proven CPA.

\section{Patients and methods}

Study design

This prospective, non-comparative trial was conducted in 18 French university hospitals between July 2005 and December 2008. Adult patients with a proven diagnosis of CPA based on a suitable chest computed tomography (CT) scan and/or a photographically documented endoscopic lesion, mycological confirmation with an Aspergillus-positive culture from a bronchopulmonary specimen, and precipitating antibodies to Aspergillus in serum ( $\geq 2$ lines) were included (the inclusion and exclusion criteria are detailed in the Supplementary Online Material, Appendix 1). All patients provided written informed consent and the protocol was approved by the centralized French ethics committee.

Patients received oral voriconazole $200 \mathrm{mg}$ (100 mg for patients weighing $<40 \mathrm{~kg}$ ) twice daily for at least 6 months, 
after a loading dose of double the maintenance dose on day 1. After this period, the investigator could continue voriconazole therapy for a maximum of six additional months if further improvement was expected. All patients were assessed for safety after 1 month. The study investigators performed efficacy (clinical, radiological, and mycological examinations) and safety evaluations every 3 months, which helped to determine treatment duration. Thus, depending on the individual patient, the treatment duration was 6 , 9, or 12 months (end of therapy, EOT); discontinuations at all other times were considered as early treatment discontinuations. Discontinuation because of treatment-related toxicity was at the discretion of the investigators. A follow-up visit was conducted 6 months after the last day of treatment.

\section{Efficacy analysis}

A data review committee consisting of the three principal investigators (J.C., B.P., P.G.) and one mycologist (C.H.) classified patients as CCPA, CNPA, or TBA based on the criteria proposed by Denning et al. [1]. They evaluated response by chest tomodensitometry or bronchoscopy (for TBA). Radiological response was defined as: (1) complete, (2) partial, (3) stabilization, or (4) failure (see Supplementary Online Material, Appendix 1). Mycological response was categorized as: (1) eradication, (2) presumed eradication (assessed at each visit separately) in patients without sputum production at any given visit, or (3) persistence (i.e., sputum or bronchopulmonary sample culture positive for $A$. fumigatus). However, due to a lack of a validated scale and inherent difficulties in distinguishing the disease under study from that of the underlying lung disease, clinical response was not included as a component of the primary endpoint.

The primary endpoint was global success at 6 months, based on previous findings and defined as complete or partial ( $\geq 50 \%$ improvement) radiological response, and mycological eradication/presumed eradication. Secondary efficacy endpoints included global success at 3, 9, and 12 months; clinical response using a $10-\mathrm{cm}$ visual analog scale for six items (cough, dyspnea, sputum, hemoptysis, chest pain, and nocturnal awakening); assessment of quality of life using the St. George's Hospital respiratory questionnaire; serologic response; all-cause mortality at end of study; and rate of CPA relapse.

\section{Safety analysis}

Vital signs and laboratory investigations (hematology, liver and renal function tests, serum chemistry, and electrocardiogram) were carried out at baseline and at every visit. Adverse events were categorized as mild, moderate, or severe, and their causality determined by the investigator.
Susceptibility testing

In vitro susceptibility testing was performed for available baseline strains of Aspergillus to amphotericin B, voriconazole, itraconazole, and posaconazole. MICs were determined using Etest strips (AB Biodisk, bioMérieux), after $48 \mathrm{~h}$ of incubation at $35{ }^{\circ} \mathrm{C}$ on RPMI 1640 medium supplemented with $2 \%$ glucose, according to the manufacturer's instructions.

The following interpretative breakpoints were used for amphotericin B [18], itraconazole, and voriconazole MIC $>2 \mathrm{mg} / \mathrm{L}$ (resistant) and for posaconazole $\mathrm{MIC}>0.5 \mathrm{mg} / \mathrm{L}$ (resistant) [19].

Serologic assessment and response

At study entry, anti-Aspergillus antibodies were detected using the screening techniques available at each site. All serum samples collected from each patient at study entry and at each study visit were sent to a centralized mycology laboratory for the detection of anti-Aspergillus antibodies by immunoelectrophoresis (further details are provided in the Supplementary Online Material, Appendix 1).

\section{Statistical analysis}

The sample size was determined based on the expected efficacy response rates; it was calculated that a sample size of 48 patients was required to demonstrate a response rate $>30 \%$, which is considered to be a positive response in phase II trials, with $80 \%$ power at a significance level of $\alpha=5 \%$. For categorical data, $95 \%$ confidence intervals (CIs) were calculated; differences were assumed as statistically significant if the upper limits for the respective $95 \%$ CIs remained below 0 . All efficacy analyses were conducted in the modified intent-to-treat population (mITT), i.e., those patients who had CPA confirmed by the data review committee, received $\geq 1$ dose of voriconazole, and underwent $\geq 1$ efficacy assessment. Data were compared between diagnoses using Pearson's $\chi^{2}$ test with $p<0.05$ indicating statistical significance. All statistical analyses were carried out using SAS software (version 8.2; SAS Institute Inc., Cary, NC).

All authors had full access to the primary data and the data analyses, and vouch for the completeness and accuracy of the data and analyses presented.

\section{Results}

Study population

The patient disposition is summarized in Fig. 1. Fifty-six patients were screened and 48 received at least one dose of 
Fig. 1 Patient disposition. IITT modified intent-to-treat; $C P A$ chronic pulmonary aspergillosis; $D R C$ data review committee; CCPA chronic cavitary pulmonary aspergillosis; $C N P A$ chronic necrotizing pulmonary aspergillosis; TBA tracheobronchial aspergillosis

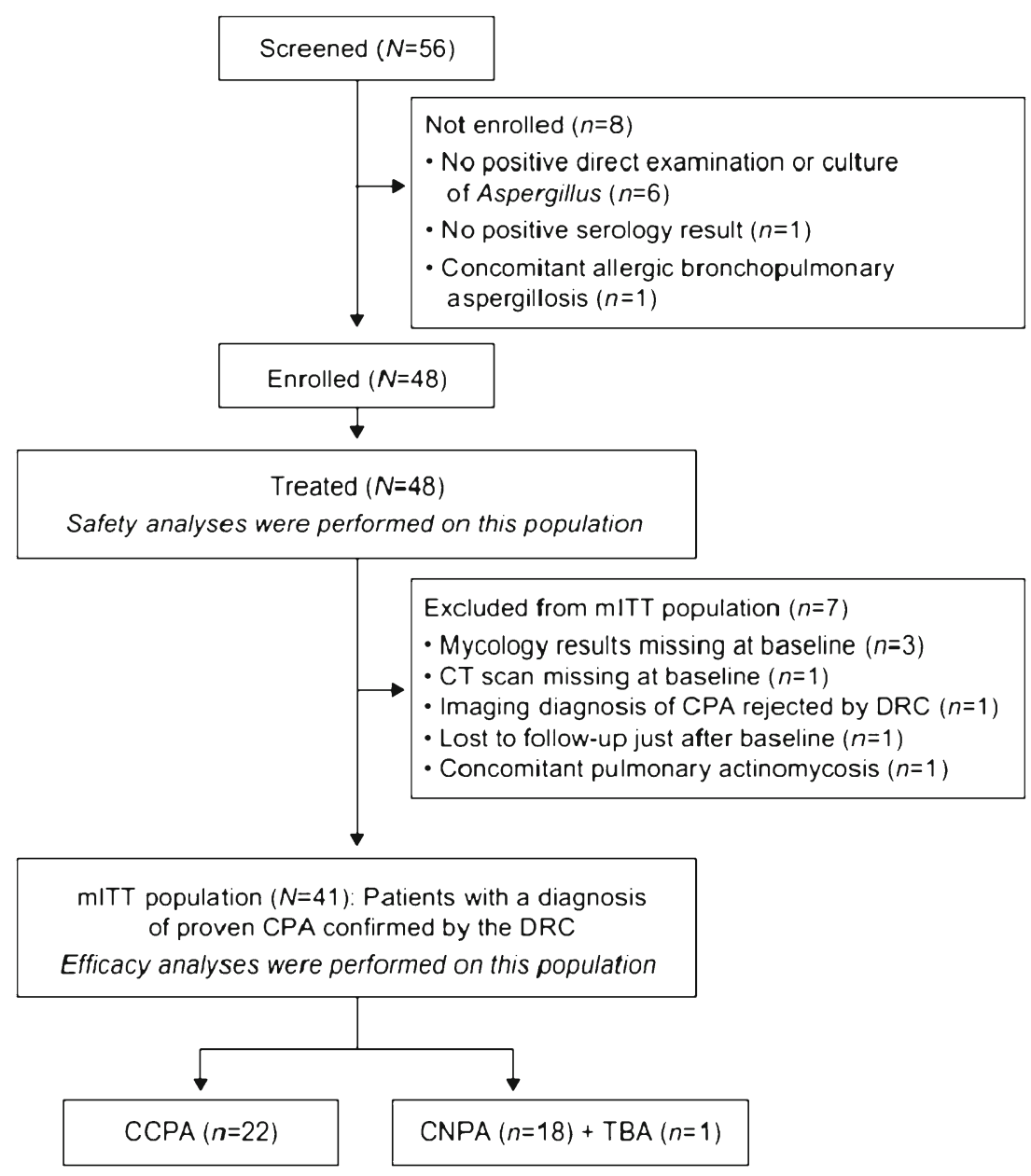

voriconazole. Of these 48 patients, 41 had confirmed CPA and comprised the mITT population. Twenty-two patients had confirmed CCPA and 19 confirmed CNPA; one patient with TBA was included into the CNPA group for all analyses, due to the clinical similarities between these two forms. A. fumigatus was isolated from bronchopulmonary specimens in all patients. At the time of inclusion, immunoelectrophoresis revealed at least two anti- $A$. fumigatus precipitin lines in all patients, with a mean of 4.9 lines (median of 4 lines; range 2-11).

The demographic and clinical characteristics are summarized in Table 1. The relatively low median body mass index of $17.3 \mathrm{~kg} / \mathrm{m}^{2}$ may reflect the overall poor health status of the study population. Among the 41 patients in the mITT population, $32(78 \%)$ continued voriconazole treatment up to 6 months and $22(54 \%)$ received voriconazole beyond 6 months. The mean treatment duration was 8.3 months (range 5 days to 13.6 months). Fifteen patients (37\%) received concomitant corticosteroid therapy (inhaled, $n=12$ and/or oral, $n=6$ ). The proportion of necrotizing and cavitary forms in these patients was similar to those patients who did not receive corticosteroids and the mITT population. Inhaled corticosteroids were started prior to the study in all patients except one, and given at the same dosage for the duration of the study. Oral corticosteroids were given for the whole study in three patients because of sarcoidosis $(n=2)$ and chronic obstructive pulmonary disease (COPD) $(n=1)$ and in one patient who received hydrocortisone substitution for adrenal insufficiency. Two other patients received one to three short courses of oral corticosteroids for bronchospasm $(n=1)$ or COPD exacerbations $(n=1)$.

Thirty-two baseline strains underwent susceptibility testing. Of these, $12 / 32$ (38\%) and 7/31 (23\%) strains exhibited resistance to amphotericin $\mathrm{B}$ and itraconazole, respectively, while a single strain was cross-resistant to itraconazole, voriconazole, and posaconazole, with MICs of $>32,16$, and $16 \mathrm{mg} / \mathrm{L}$, respectively (Table 2).

Efficacy analyses

Global success

Global success at 6 months, the primary endpoint of this study, was reported in 13/41 (32\%) patients (95\% CI, 18.1$48.1 \%)$ : 10/19 (53\%) with CNPA and 3/22 (14\%) with CCPA $(p=0.01)$ (Fig. 2). 
Table 1 Demographic and clinical characteristics of patients in the modified intent-to-treat population (mITT) $(N=41)^{\mathrm{a}}$

Demographic characteristics

Male, $n(\%)$

$24(58.5)$

Median age at baseline, years (range)

$58.0(25.7-81.6)$

Median BMI, $\mathrm{kg} / \mathrm{m}^{2}$ (range)

$17.3(13.2-38.9)$

Underlying lung disease/condition ${ }^{\mathrm{b}}, n(\%)$

COPD

18 (43.9)

Prior tuberculosis or mycobacteriosis

$13(31.7)$

Bronchiectasis

$6(14.6)$

Pneumothorax

$5(12.2)$

Prior lung cancer

$3(7.3)$

Sarcoidosis

$3(7.3)$

$3(7.3)$

$2(4.9)$

$1(2.4)$

Aspergilloma surgery

11 (26.8)

Other

15 (36.6)

Corticosteroids ${ }^{c}$

12

Inhaled

Systemic

$4(9.8)$

2 (4.9)

$11(26.8)$

Diabetes mellitus

$12(29.3)$

None identified

$B M I$ body mass index; $C O P D$ chronic obstructive pulmonary disease

${ }^{\mathrm{a}}$ The mean number of patients recruited per centre was 2.3 (median 2; range $1-5)$

${ }^{\mathrm{b}}$ Some patients had two or more underlying diseases/conditions

${ }^{\mathrm{c}}$ Three patients received both inhaled and systemic corticosteroids

The rate of success was similar after 3 months of treatment for CNPA ( $53 \%$ ) but lower for CCPA (9\%). Global success at EOT was reported in 18/41 (44 \%) patients: $11 / 19(58 \%)$ with CNPA and 7/22 (32\%) with CCPA ( $p=0.09)$ (Fig. 2). The mean duration of treatment for patients with global success at EOT did not differ between patients with CNPA (10 months; range: 6-13.4) and CCPA (11 months; range: 6.6-12.5).

The success rate at 6 months in patients receiving concomitant corticosteroids $(20 \%)$ was lower than in patients who were not receiving concomitant corticosteroids (38\%).

Table 2 In vitro susceptibility testing of Aspergillus strains collected at baseline

\begin{tabular}{lllll}
\hline & $\mathrm{MIC}_{50}$ & $\mathrm{MIC}_{90}$ & $\mathrm{GM}$ & Range $(\mathrm{mg} / \mathrm{L})$ \\
\hline Amphotericin B $(n=32)$ & 1.50 & 4.00 & 1.17 & $0.09-32.00$ \\
Itraconazole $(n=31)$ & 1.50 & 6.00 & 1.32 & $0.05-32.00$ \\
Posaconazole $(n=32)$ & 0.094 & 0.19 & 0.12 & $0.03-16.00$ \\
Voriconazole $(n=32)$ & 0.125 & 0.25 & 0.14 & $0.05-16.00$ \\
\hline
\end{tabular}

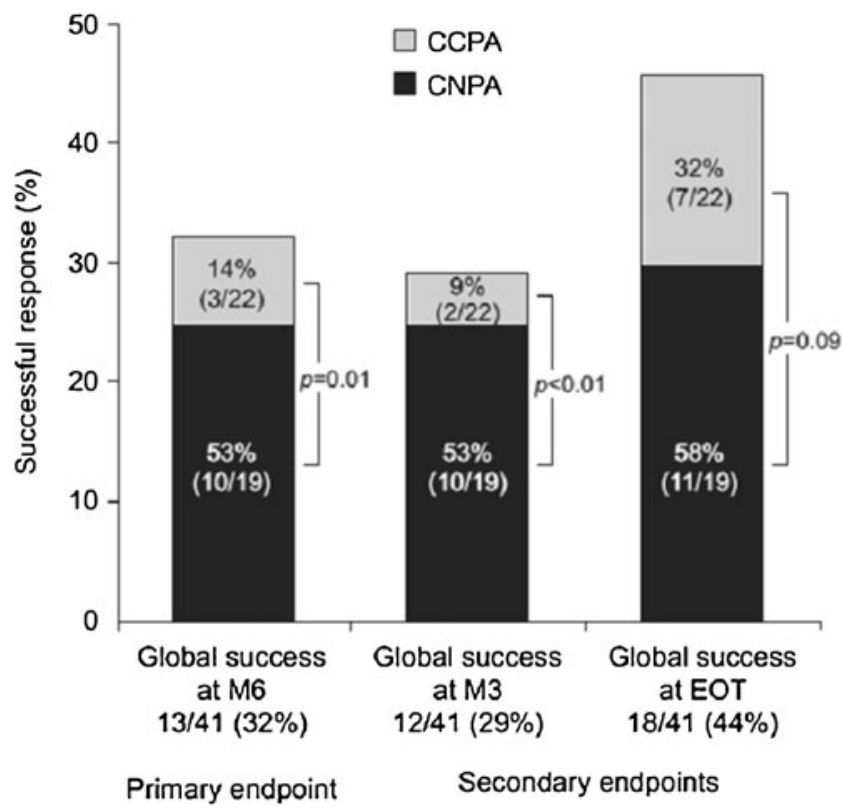

Fig. 2 Global success of voriconazole therapy at 3 months, 6 months, and end of treatment. Global success was defined as a complete or partial ( $\geq 50 \%$ improvement) radiological response and mycological eradication/presumed eradication. CCPA chronic cavitary pulmonary aspergillosis; CNPA chronic necrotizing pulmonary aspergillosis; M3 3-month visit; M6 6-month visit; EOT end of treatment

\section{Radiological response}

Radiological response was evaluated at EOT for 31 patients (Figs. 3 and 4). CPA was considered as controlled (complete or partial response or stabilization) in all cases, with the exception of one patient with CCPA $(30 / 31 ; 97 \%)$. Of the radiologically evaluable patients, $41 \%$ with CCPA showed

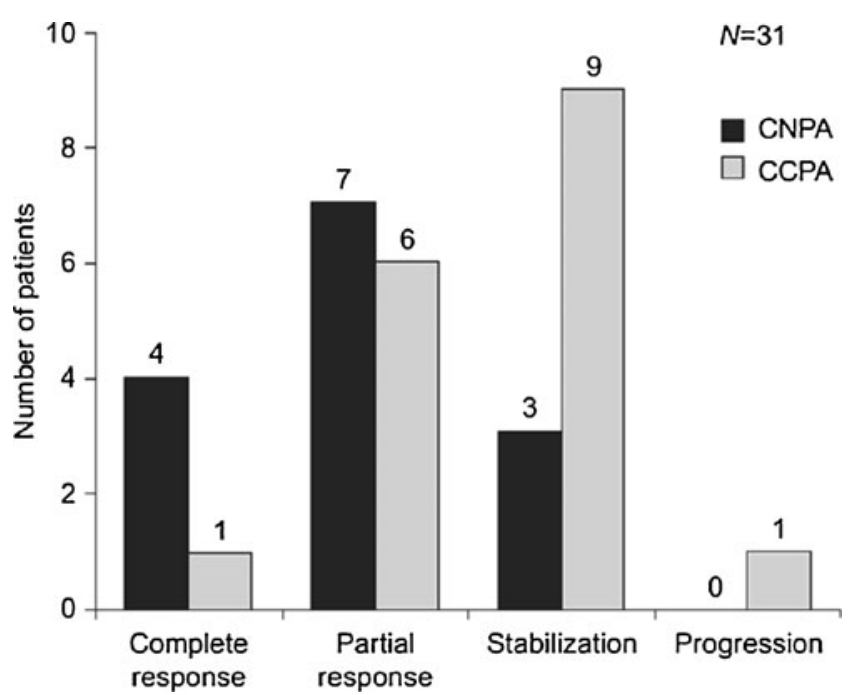

Fig. 3 Radiological response at end of treatment (defined as the last available radiological response for each patient receiving $\geq 6$ months of treatment). CCPA chronic cavitary pulmonary aspergillosis; $C N P A$ chronic necrotizing pulmonary aspergillosis 
Fig. 4 Examples of

radiological response in chronic pulmonary aspergillosis treated with voriconazole. Chest computed tomography (CT) scan of a patient with chronic cavitary pulmonary aspergillosis (CCPA) at baseline (a) and after 6 months of treatment (b), indicating partial regression of the mycetoma (asterisk) and of the adjacent pleural thickening (arrow). Chest CT scan of a patient with chronic necrotizing pulmonary aspergillosis

(CNPA) at baseline (c) and after 6 months of treatment (d), indicating total disappearance of the left upper lobe focal consolidation
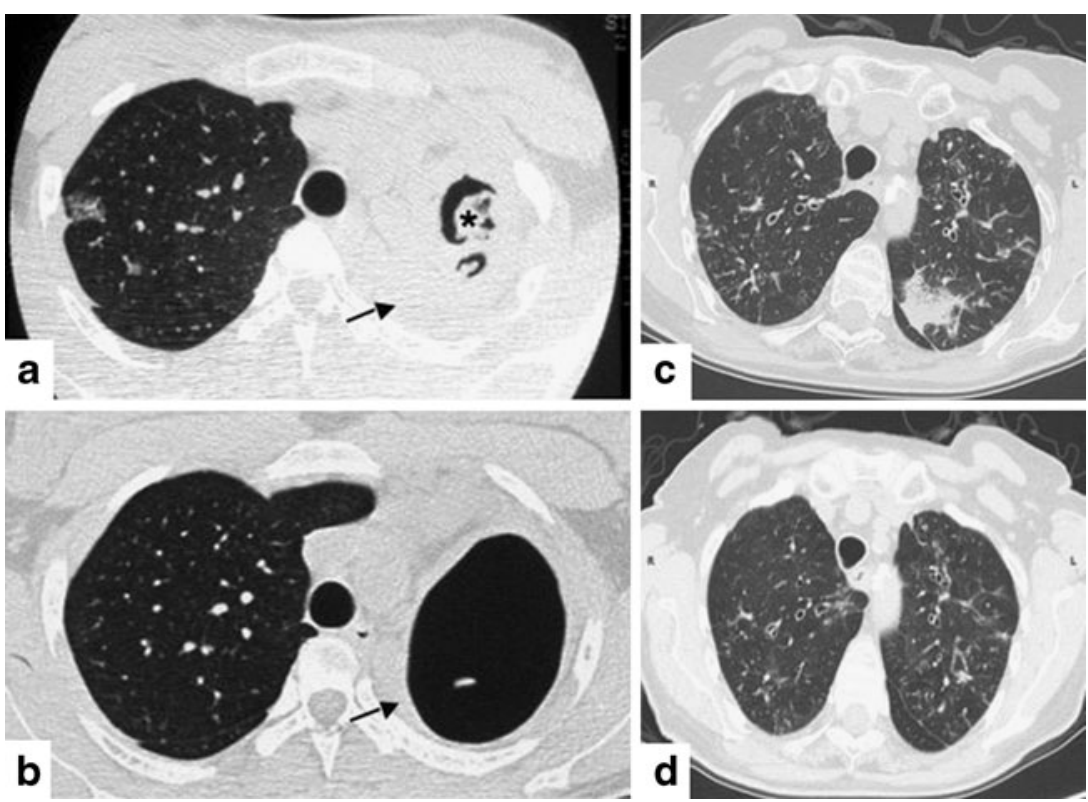

a complete or partial response and $53 \%$ stabilization; $79 \%$ with CNPA had a complete or partial response and $21 \%$ stabilized with voriconazole treatment (Fig. 3).

\section{Clinical response}

Improvements in global respiratory symptoms were seen at 6 and 12 months (Fig. 5). A significant improvement in quality of life was noted at 6 months and at the end of study (Supplementary Online Material, Table 1).

\section{Mycological response}

Evaluation of the mycological response revealed eradication or presumed eradication of A. fumigatus from the bronchopulmonary specimens of all patients at both 6 months and at EOT. Eradications at 6 months were documented in 26 of $32(81 \%)$ patients who received study treatment for up to 6 months, and at EOT in 18 of $22(82 \%)$ patients who continued study treatment beyond 6 months. The patient with the pan-azole-resistant strain had CCPA with a presumed eradication at 6 months and EOT, and was considered a failure at both timepoints because of an insufficient radiological response.

At 6 months, 8/29 (28\%) patients had negative serology and $14(48 \%)$ showed a marked decrease in their specific immunologic response (decrease of $\geq 2$ lines). All patients with CNPA showed improvement compared to $64 \%$ of patients with CCPA. Overall, the mean number of Aspergillus precipitin lines decreased from 4.9 at baseline to 3.3 at

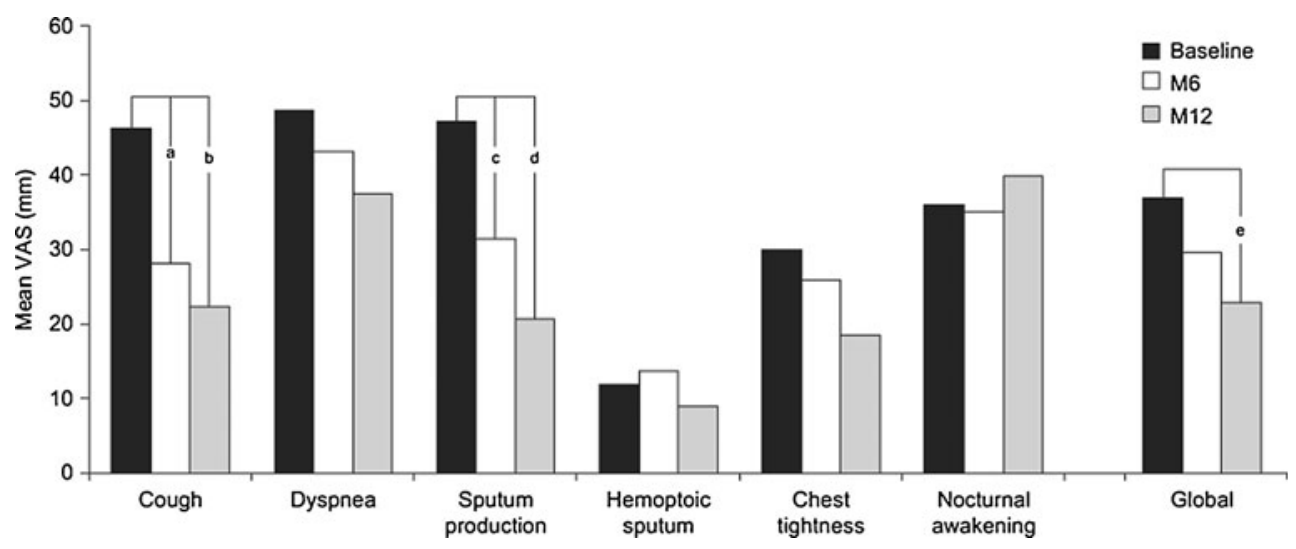

Fig. 5 Clinical symptoms at 6 months (MO) and 12 months (M12) measured by a $10-\mathrm{cm}$ visual analog scale (VAS). Significant decreases were observed at 6 months for cough $\left({ }^{\mathrm{a}}-19.6 \mathrm{~mm} ; 95 \% \mathrm{CI},-33.7\right.$ to -5.6 ) and sputum production ( ${ }^{\mathrm{c}}-14.9 \mathrm{~mm} ; 95 \% \mathrm{CI},-29.3$ to -0.5 ), and at 12 months for cough $\left({ }^{b}-30.1 \mathrm{~mm} ; 95 \% \mathrm{CI},-49.9\right.$ to -10.4$)$, sputum production ( ${ }^{\mathrm{d}}-26.5 \mathrm{~mm} ; 95 \% \mathrm{CI},-40.1$ to -12.8$)$, and mean global VAS ( ${ }^{\mathrm{e}}-15.9 \mathrm{~mm}$; $95 \% \mathrm{CI},-27.3$ to -4.4 ) 
6 months. However, there was no difference in the respective results between patients with and without global success (Supplementary Online Material, Table 2).

Radiological or mycological relapse was observed at the 6-month post-therapeutic visit in 3/18 (17\%) patients (one each with CCPA, CNPA, and TBA) who had previously experienced global success at their respective EOT visits.

\section{Safety analysis}

Safety was assessed in all patients who received at least one dose of voriconazole $(n=48)$. Side effects were consistent with the known adverse-event profile of voriconazole [20]: patients reported visual disturbances $(21 \%)$; photosensitivity reactions (19\%); blurred vision (12\%); constipation, vomiting, and $\gamma$-glutamyl-transferase increase (10\% each); chills, decreased appetite, headache, and insomnia (8\% each); and vertigo, nausea, cholestasis, weight loss, and anorexia (6 \% each). Seven patients discontinued voriconazole for toxicity, i.e., grade 3 liver toxicity $(n=2)$, grade 1 hyponatremia, QT prolongation ( $80 \mathrm{~ms}$ increase), memory disorder, insomnia and memory loss, or worsening of general condition ( $n=1$ each). No death was attributable to drug toxicity. Five patients died during the study from comorbidities (bacterial pneumonia, pneumothorax, chronic respiratory failure, ovarian cancer, or septic shock); no deaths were attributable to CPA in this study.

\section{Discussion}

In our study, voriconazole was found to be effective as a primary therapy for CPA; global success was observed in 32 and $44 \%$ of patients at 6 months and at $\geq 6$ months of treatment, respectively. CPA was controlled according to radiological assessments in $97 \%$ of patients, i.e., in all those with CNPA and $94 \%$ of those with CCPA. There were also statistically significant improvements in some CPA-related symptoms (cough and sputum production), which may more closely reflect improvements in the inflammatory processes due to Aspergillus infection.

This is the first study of antifungal therapy for CPA with an extensive follow-up period to demonstrate a quality-oflife improvement in patients with severe underlying lung diseases and comorbid conditions. Prolonged voriconazole therapy was well tolerated, with adverse events either related to the underlying pathology or consistent with the known side-effect profile of voriconazole [20]. Photosensitivity reactions occurred in almost $20 \%$ of patients in this study.

The global success rates for CPA were similar at $3(29 \%)$ and $6(32 \%)$ months following treatment with voriconazole, suggesting that treatment for 3 months could be sufficient for some patients, especially those with CNPA. Indeed, treatment outcome was more frequently favorable and achieved more rapidly in patients with necrotizing forms of CPA than in those with CCPA. This could be due to the fungus having been more exposed to voriconazole in CNPA than CCPA. Aspergillosis infection develops within parenchymal lung tissue in CNPA, whereas it exists within a cavity surrounded by fibrotic tissue in CCPA [1], thus potentially hindering the penetration of antifungals into the site of infection. The marked increase in response rate among CCPA patients from the 6-month visit to EOT suggests that a longer duration of treatment ( $>6$ months) may be necessary in patients with cavitary forms of CPA.

Three relapses were observed at the 6-month follow-up visit, one each in patients with CNPA, CCPA, and TBA. In the CNPA patient, fungal isolates obtained at initial diagnosis and at relapse were different as judged by multilocus microsatellite analysis (data not shown) [21]. This suggests that the relapse was due to reinfection with a new strain and not reactivation of the primary infection.

At baseline, only one strain was found to exhibit panazole resistance (i.e., including voriconazole) among the 32 strains tested. In contrast, resistance to itraconazole ( $23 \%)$ and amphotericin B (38\%) were common. The high number of amphotericin B-resistant strains was unexpected. This patient population is rarely treated with amphotericin $B$ and no previous treatment with amphotericin B was reported in the present study population, although we cannot exclude this possibility with complete certainty. It has been suggested that the Etest may be better able to differentiate between amphotericin B-susceptible and -resistant strains than traditional broth microdilution methods [22]. This may explain, at least in part, the high number of resistant strains observed in the present study.

Both resistance to antifungals and pre-existing pulmonary disease may have been important determinants of the overall outcome in our study. As mentioned above, it was not possible to evaluate the effect of antifungal resistance, as there was only one strain with an elevated MIC to voriconazole. Underlying pulmonary diseases were inherent to our study population and the outcome could have been influenced by the progression of the underlying pulmonary condition rather than therapeutic failure. Other factors may also have influenced outcome, such as concomitant corticosteroid therapy, as shown by the lower success rate in these patients.

Our findings are consistent with other published observations of oral voriconazole used in this setting [15-17] and support the current recommendations of the Infectious Diseases Society of America (IDSA), which give voriconazole a $\mathrm{B}$ grading for CPA therapy in general and CNPA in particular [23]. Our efficacy results compare favorably, in terms of treatment success, relapse rate, and radiological response, with those from prospective clinical trials with 
oral itraconazole for CNPA and aspergilloma [6, 24]. However, unlike our own study, these trials classified CPA disease stabilization as a treatment success, which further underscores the potential efficacy differences between voriconazole and itraconazole. Furthermore, diagnostic criteria, evaluation of efficacy, and study endpoints were not clearly defined in the itraconazole studies [6, 24].

The other antifungals evaluated in this setting were oral posaconazole and intravenous micafungin [25-28]; data with intravenous amphotericin $\mathrm{B}$ are available, but are sparse and discouraging. In a retrospective case series, posaconazole exhibited a response rate of 61 and $46 \%$ at 6 and 12 months, respectively, where a successful response was defined as no clinical and/or radiological deterioration, and, as such, included stable disease [25]. Micafungin was evaluated in a retrospective case series, a prospective, noncomparative study, and a comparative trial with voriconazole [26-28]. Although these studies suggest good tolerability and efficacy of micafungin in the treatment of CPA, the results need to be interpreted with caution. For instance, treatment duration was short (maximum of 8 weeks in the open-label study and 4 weeks in the comparative study). Furthermore, treatment success was defined by subjective criteria, such as clinical improvement, decreased plasma levels of inflammatory markers, and "...apparent improvement in the newly appeared lesions on radiological images" [26-28].

Our trial had some limitations which should be considered when interpreting the study findings. A comparator or control group was not included in our study. Our study also did not include therapeutic drug monitoring or long-term data on relapses/recurrences or survival status beyond the 6month follow-up period. However, the study had several strengths compared with previous studies of voriconazole and other antifungals in this setting, such as the prospective study design, efficacy assessment based on imaging confirmed by a data review committee, treatment duration of 612 months, and a definition of global success that included only complete and partial radiological responses.

This is the first prospective, multicenter study with a central review of predefined radiological response criteria to demonstrate the effectiveness of voriconazole therapy in patients with CPA, particularly those with CNPA. Favorable treatment response was observed from 3 months onwards in CNPA patients, but improvement required at least 612 months in CCPA patients. Future studies should evaluate the value of voriconazole dose adjustments based on plasma levels, an approach associated with potential improvements in the efficacy and safety of voriconazole for invasive aspergillosis [29].

Acknowledgments This study was sponsored by Pfizer. The authors would like to thank Sophie Thorez, Sandra Vellaissamy, and
Dominique Rocherolle, technical assistants at APHP, Hôpital SaintAntoine, Parasitology-Mycology Laboratory. Editorial support was provided by Dominik Wolf at PAREXEL and was funded by Pfizer International Operations. Statistical support was provided by Fiona Brock and Peter Cooper (Quanticate; funded by Pfizer) and Paul J. Miller (Pfizer).

Study collaborators In addition to the named authors, the following investigators participated in this study: C. Bernier, Hôpital René Pleven, Dinan; F. Couturaud, Hôpital Cavale Blanche, Brest; B. Crestani, Hôpital Bichat, Paris; C. Darne, Centre Medical de Bligny, Bris sous Forges; S. Dominique, Hôpital Germont, Rouen; F. Lebargy, Hôpital Maison Blanche, Reims; C. Pison, Hôpital A. Michallon, Grenoble; I. Tillie-Leblond, Hôpital A. Calmette, Lille; and D. Valeyre, Hôpital Avicenne, Bobigny.

Author contributions Guarantor for the entire manuscript: J.C. Study design (conception): J.C., M.P.

Study design (review): B.P., P.G., C.H.

Principal investigator of the study: J.C.

Recruited patients (i.e., data collection): J.C., B.P., P.G., A.B., E.B., A.B., V.C., T.J., C.G.

Data analysis/interpretation: J.C., C.H., B.P., P.G., M.P. (A.B., E.B., A.B., V.C., T.J., C.G. also reviewed the overall results).

Principally responsible for manuscript writing: J.C.

Critically reviewed/revised manuscript for all content: all authors.

Approved final version of the submitted manuscript: all authors.

Conflicts of interest This study, initially proposed by J.C., was designed by the academic authors (J.C., P.G., B.P., and C.H.), along with employees of the sponsor. Employees of the sponsor were also involved in the analysis and interpretation of the data, and one author (M.P.) is a full-time employee of the sponsor. All authors had full access to the primary data and the data analyses, and vouch for the completeness and accuracy of the data and analyses presented. The authors report the following conflicts of interest: J.C. received speaker and consultancy fees from Pfizer; B.P. received consultancy fees from Pfizer; C.H. received research grants from MSD and Pfizer, travel support from Astellas, Pfizer, Gilead, MSD, and Schering-Plough, speaker fees from MSD, Pfizer, and Schering-Plough, and consultancy fees from MSD and Pfizer; A. Bergeron received speaker fees from Pfizer and Schering-Plough, consultancy fees from Schering-Plough, and travel support from Gilead and Pfizer; V.C. received speaking fees from Pfizer; E.B., A. Bourdin, T.J., and C.G. do not have any financial relationships with a commercial entity that has an interest in the subject of this manuscript; M.P. is a full-time employee of Pfizer; P.G. received consultancy fees from Pfizer.

\section{References}

1. Denning DW, Riniotis K, Dobrashian R, Sambatakou H (2003) Chronic cavitary and fibrosing pulmonary and pleural aspergillosis: case series, proposed nomenclature change, and review. Clin Infect Dis 37(Suppl 3):S265-S280

2. Smith NL, Denning DW (2011) Underlying conditions in chronic pulmonary aspergillosis including simple aspergilloma. Eur Respir J 37:865-872

3. Soubani AO, Chandrasekar PH (2002) The clinical spectrum of pulmonary aspergillosis. Chest 121:1988-1999

4. Uffredi ML, Mangiapan G, Cadranel J, Kac G (2003) Significance of Aspergillus fumigatus isolation from respiratory specimens of 
nongranulocytopenic patients. Eur J Clin Microbiol Infect Dis 22:457-462

5. Saraceno JL, Phelps DT, Ferro TJ, Futerfas R, Schwartz DB (1997) Chronic necrotizing pulmonary aspergillosis: approach to management. Chest 112:541-548

6. Dupont B (1990) Itraconazole therapy in aspergillosis: study in 49 patients. J Am Acad Dermatol 23:607-614

7. Denning DW, Ribaud P, Milpied N, Caillot D, Herbrecht R, Thiel E et al (2002) Efficacy and safety of voriconazole in the treatment of acute invasive aspergillosis. Clin Infect Dis 34:563-571

8. Herbrecht R, Denning DW, Patterson TF, Bennett JE, Greene RE, Oestmann JW et al; Invasive Fungal Infections Group of the European Organisation for Research and Treatment of Cancer and the Global Aspergillus Study Group (2002) Voriconazole versus amphotericin B for primary therapy of invasive aspergillosis. N Engl J Med 347:408-415

9. Perfect JR, Marr KA, Walsh TJ, Greenberg RN, DuPont B, de la Torre-Cisneros $\mathrm{J}$ et al (2003) Voriconazole treatment for lesscommon, emerging, or refractory fungal infections. Clin Infect Dis 36:1122-1131

10. Jacobs F, Selleslag D, Aoun M, Sonet A, Gadisseur A (2011) An observational efficacy and safety analysis of the treatment of acute invasive aspergillosis using voriconazole. Eur J Clin Microbiol Infect Dis 31:1173-1179

11. Pfaller MA, Messer SA, Hollis RJ, Jones RN; SENTRY Participants Group (2002) Antifungal activities of posaconazole, ravuconazole, and voriconazole compared to those of itraconazole and amphotericin B against 239 clinical isolates of Aspergillus spp. and other filamentous fungi: report from SENTRY Antimicrobial Surveillance Program, 2000. Antimicrob Agents Chemother 46:1032-1037

12. Herbrecht R (2004) Voriconazole: therapeutic review of a new azole antifungal. Expert Rev Anti Infect Ther 2:485-497

13. Krishnan S, Manavathu EK, Chandrasekar PH (2005) A comparative study of fungicidal activities of voriconazole and amphotericin B against hyphae of Aspergillus fumigatus. J Antimicrob Chemother 55:914-920

14. Capitano B, Potoski BA, Husain S, Zhang S, Paterson DL, Studer SM et al (2006) Intrapulmonary penetration of voriconazole in patients receiving an oral prophylactic regimen. Antimicrob Agents Chemother 50:1878-1880

15. Sambatakou H, Dupont B, Lode H, Denning DW (2006) Voriconazole treatment for subacute invasive and chronic pulmonary aspergillosis. Am J Med 119:527.e17-527.e24

16. Jain LR, Denning DW (2006) The efficacy and tolerability of voriconazole in the treatment of chronic cavitary pulmonary aspergillosis. J Infect 52:e133-e137

17. Camuset J, Nunes H, Dombret MC, Bergeron A, Henno P, Philippe B et al (2007) Treatment of chronic pulmonary aspergillosis by voriconazole in nonimmunocompromised patients. Chest 131:1435-1441

18. European Committee on Antimicrobial Susceptibility Testing (EUCAST) (2012) Amphotericin B and Aspergillus spp.: rationale for the EUCAST clinical breakpoints, v1.0, January 2012. http:// www.eucast.org. Accessed 24 May 2012

19. Verweij PE, Howard SJ, Melchers WJ, Denning DW (2009) Azole-resistance in Aspergillus: proposed nomenclature and breakpoints. Drug Resist Updat 12:141-147

20. European Medicines Agency (EMA) (2009) Vfend: summary of product characteristics (revision 17). Available online at: http:// www.ema.europa.eu/humandocs/Humans/EPAR/vfend/vfend.htm. Accessed 9 September 2011

21. Bart-Delabesse E, Humbert JF, Delabesse E, Bretagne S (1998) Microsatellite markers for typing Aspergillus fumigatus isolates. $\mathrm{J}$ Clin Microbiol 36:2413-2418

22. Colozza C, Posteraro B, Santilli S, De Carolis E, Sanguinetti M, Girmenia C (2012) In vitro activities of amphotericin B and AmBisome against Aspergillus isolates recovered from Italian patients treated for haematological malignancies. Int J Antimicrob Agents 39:440-443

23. Walsh TJ, Anaissie EJ, Denning DW, Herbrecht R, Kontoyiannis DP, Marr KA et al; Infectious Diseases Society of America (2008) Treatment of aspergillosis: clinical practice guidelines of the Infectious Diseases Society of America. Clin Infect Dis 46:327-360

24. De Beule K, De Doncker P, Cauwenbergh G, Koster M, Legendre $\mathrm{R}$, Blatchford $\mathrm{N}$ et al (1988) The treatment of aspergillosis and aspergilloma with itraconazole, clinical results of an open international study (1982-1987). Mycoses 31:476-485

25. Felton TW, Baxter C, Moore CB, Roberts SA, Hope WW, Denning DW (2010) Efficacy and safety of posaconazole for chronic pulmonary aspergillosis. Clin Infect Dis 51:1383-1391

26. Kohno S, Izumikawa K, Ogawa K, Kurashima A, Okimoto N, Amitani R et al; Japan Chronic Pulmonary Aspergillosis Study Group (JCPASG) et al (2010) Intravenous micafungin versus voriconazole for chronic pulmonary aspergillosis: a multicenter trial in Japan. J Infect 61:410-418

27. Izumikawa K, Ohtsu Y, Kawabata M, Takaya H, Miyamoto A, Sakamoto $\mathrm{S}$ et al (2007) Clinical efficacy of micafungin for chronic pulmonary aspergillosis. Med Mycol 45:273-278

28. Kohno S, Masaoka T, Yamaguchi $\mathrm{H}$, Mori T, Urabe A, Ito A et al (2004) A multicenter, open-label clinical study of micafungin (FK463) in the treatment of deep-seated mycosis in Japan. Scand J Infect Dis 36:372-379

29. Pascual A, Calandra T, Bolay S, Buclin T, Bille J, Marchetti O (2008) Voriconazole therapeutic drug monitoring in patients with invasive mycoses improves efficacy and safety outcomes. Clin Infect Dis 46:201-211 